\title{
Cortical cerebral blood flow in ageing: effects of haematocrit, sex, ethnicity and diabetes
}

\author{
Lorna A. Smith ${ }^{1,2}$ (D) Andrew Melbourne ${ }^{3,4} \cdot$ David Owen $^{3,4} \cdot$ M. Jorge Cardoso ${ }^{3,5} \cdot$ Carole H. Sudre $^{3,4,5} \cdot$ Therese Tillin $^{1}$ • \\ Magdalena Sokolska ${ }^{6}$ - David Atkinson ${ }^{2} \cdot$ Nish Chaturvedi $^{1} \cdot$ Sebastien Ourselin $^{3}$ - Alun D. Hughes ${ }^{1}$. \\ Frederik Barkhof $^{4,5,7} \cdot$ Hans Rolf Jäger ${ }^{8,9}$
}

Received: 24 July 2018 / Revised: 24 December 2018 / Accepted: 11 February 2019 / Published online: 18 March 2019

(C) The Author(s) 2019

\begin{abstract}
Objectives Cerebral blood flow (CBF) estimates from arterial spin labelling (ASL) show unexplained variability in older populations. We studied the impact of variation of haematocrit (Hct) on CBF estimates in a tri-ethnic elderly population.

Materials and methods Approval for the study was obtained from the Fulham Research Ethics Committee and participants gave written informed consent. Pseudo-continuous arterial spin labelling was performed on 493 subjects (age 55-90) from a tri-ethnic community-based cohort recruited in London. CBF was estimated using a simplified Buxton equation, with and without correction for Hct measured from blood samples. Differences in perfusion were compared, stratified by sex, ethnicity and diabetes. Results of Student's $t$ tests were reported with effect size.

Results Hct adjustment decreased CBF estimates in all categories except white European men. The decrease for women was 2.7 $(3.0,2.4) \mathrm{mL} / 100 \mathrm{~g} / \mathrm{min}$ ) (mean (95\% confidence interval (CI)), $p<0.001 d=0.38$. The effect size differed by ethnicity with estimated mean perfusion in South Asian and African Caribbean women found to be lower by $3.0(3.6,2.5) \mathrm{mL} / 100 \mathrm{~g} / \mathrm{min}$, $p<0.001 d=0.56$ and $3.1(3.6,2.5) \mathrm{mL} / 100 \mathrm{~g} / \mathrm{min}), p<0.001 d=0.48$, respectively. Estimates of perfusion in subjects with diabetes decreased by $1.8(2.3,1.4) \mathrm{mL} / 100 \mathrm{~g} / \mathrm{min}, p<0.001 d=0.23)$ following Hct correction. Correction for individual Hct altered sample frequency distributions of CBF values, especially in women of non-European ethnicity.

Conclusion ASL-derived CBF values in women, non-European ethnicities and individuals with diabetes are overestimated if calculations are not appropriately adjusted for individual Hct.
\end{abstract}

Electronic supplementary material The online version of this article (https://doi.org/10.1007/s00330-019-06096-w) contains supplementary material, which is available to authorized users.

Lorna A. Smith

lorna.smith.13@ucl.ac.uk

1 MRC Unit for Lifelong Health and Ageing, Department of Population Science \& Experimental Medicine, University College London, WC1E 6HX, London, UK

2 Centre for Medical Imaging, Division of Medicine, University College London, 2nd Floor, Charles Bell House, 43-45 Foley Street, London W1W 7TS, UK

3 School of Biomedical Engineering and Imaging Sciences, King's College London, London SE1 7EH, UK

4 Department of Medical Physics and Biomedical Engineering, University College London, London NW1 2BU, UK
5 Dementia Research Centre, UCL Institute of Neurology, London Wc1N 3BG, UK

6 Institute of Healthcare Engineering, University College London, London, UK

7 Department of Radiology \& Nuclear Medicine, VU University Medical Centre, Amsterdam, Netherlands

8 Department of Brain Repair and Rehabilitation, UCL Institute of Neurology, London WC1N 3BG, UK

9 Lysholm Department of Neuroradiology, The National Hospital for Neurology and Neurosurgery, University College London, London WCN1 3BG, UK 


\section{Key Points}

- $C B F$ quantification from ASL using a fixed Hct of 43.5\%, as recommended in the ISMRM white paper, may lead to erroneous CBF estimations particularly in non-European and female subjects.

- Individually measured Hct values improve the accuracy of CBF estimation and, if these are not available, an adjusted value according to gender, ethnicity or diabetes status should be considered.

- Hct-corrected ASL could be potentially important for CBF threshold decision making in the fields of neurodegenerative disease and neuro-oncology.

Keywords Cerebrovascular circulation · Haematocrit · Ageing · Ethnic groups

$\begin{array}{ll}\begin{array}{l}\text { Abbreviations } \\ \text { ASL }\end{array} & \text { Arterial spin labelling } \\ \text { BMI } & \text { Body mass index } \\ \text { CBF } & \text { Cerebral blood flow } \\ \mathrm{CMRO}_{2} & \text { Cerebral metabolic rate of oxygen } \\ \mathrm{HbAlc} & \text { Haemoglobin Alc (glycated } \\ & \text { haemoglobin) } \\ \mathrm{Hct} & \text { Haematocrit } \\ \mathrm{HDL} \text { cholesterol } & \text { High-density lipoprotein cholesterol } \\ \text { LDL cholesterol } & \text { Low-density lipoprotein cholesterol } \\ \text { PCASL } & \text { Pseudo-continuous arterial spin labelling } \\ \text { PLD } & \text { Post-labelling delay } \\ \text { PVC } & \text { Partial volume correction }\end{array}$

\section{Introduction}

Arterial spin labelling (ASL) is a magnetic resonance imaging (MRI) technique increasingly used in research and clinical settings to calculate cerebral blood flow (CBF) noninvasively [1]. It has been recognised that ASL can provide an early biomarker for dementia, cognitive decline and small vessel disease [2-8]. Despite statistical differences between groups, e.g. Alzheimer's disease, mild cognitive impairment and normal ageing, clinical applications have been hampered by unexplained inter-subject variability $[9,10]$.

Deriving quantitative perfusion values from the raw MRI signal requires the application of a model containing several assumptions that relate to physiological properties of the blood and tissues. The white paper recommendations of the International Society for Magnetic Resonance in Medicine (ISMRM) and the European Consortium for ASL in Dementia propose pseudo-continuous ASL (PCASL) with a single post-labelling delay (PLD) and slice-timing correction, and the application of a simplified Buxton equation for quantification of CBF $[11,12]$. In this model, $1650 \mathrm{~ms}$ is recommended as the longitudinal relaxation time of blood $\left(\mathrm{T}_{\mathrm{blood}}\right)$ at $3 \mathrm{~T}$. This has been derived from a linear relationship between haematocrit (Hct) and blood T1 found in experiments under appropriate physiological conditions [13], assuming an average adult Hct of $43.5 \%$ [11]. However, there are well-recognised sources of variation in Hct between and within populations that may render $\mathrm{CBF}$ estimation inaccurate if they are excluded from the model $[9,10]$.

Hct varies by sex with females typically having lower Hct than males [14]. Most studies show a lower Hct in blacks than Asians compared with whites $[15,16]$, although an earlier study found this varied according to age and was not applicable in some younger subjects (men aged 15-24) [17]. Higher Hct levels have been associated with obesity [14] and have also been associated with risk of developing diabetes [18]. Diabetic patients with long-standing disease may have decreased Hct, possibly due to diabetic nephropathy causing erythropoietin deficiency [19], or malabsorption of vitamin $\mathrm{B}_{12}$ as a side-effect of long-term treatment with metformin [20].

The purpose of this study was to identify the variability in cortical CBF using ASL, to investigate the influence of Hct on the estimation of $\mathrm{CBF}$ and to determine how this impacts on the associations of CBF with age, sex, ethnicity and diabetes.

\section{Materials and methods}

\section{Study population}

Subjects ( $n=493,40 \%$ female, age range 55-90) were recruited from the Southall and Brent Revisited (SABRE) study. Approval for investigations was obtained from the Fulham Research Ethics Committee (ref: 14/LO/0108) and participants gave written informed consent. SABRE is a longitudinal study principally investigating cardio-metabolic disease in a tri-ethnic population cohort; details have been published elsewhere [21]. Briefly, participants were community-dwelling elderly men and women and resident in the north and northwest London at the commencement of the SABRE study in 1988. Ethnicity was defined based on the country of origin of all four grandparents. Spouses or significant others were invited to participate in a clinical visit, commencing in 2014. An additional booster sample of African Caribbeans from the same area of London was recruited to increase numbers and therefore enhance the power of the study. Data for this study were acquired between August 2014 and October 2016. 


\section{Investigations}

Participants underwent all examinations on the same day as MRI. Participants were requested to consume no more than an early light breakfast prior to arrival at the clinic. Weight and height were measured using a Tanita Pro BC-418 Body Composition Analyser and a Seca 216 Stadiometer, respectively, and body mass index (BMI) was calculated. Supine central blood pressure (BP) and heart rate (HR) were measured using a Pulsecor device after 5-min rest, in both arms. BP was calculated as the average of the final two of three measurements in the left arm unless the difference between arms was $>10 \mathrm{mmHg}$ in which case the arm with the higher BP was used. Mean arterial pressure (MAP) was calculated using a Pulsecor device (www.uscom.com.au) to measure the arterial pressure waveform during suprasystolic brachial cuff inflations. Hct was measured using an impedance-based, direct current sheath flow method (Sysmex XE-2100) from a venous blood sample drawn on the same morning as the MRI examination. Blood was also analysed for glycosylated haemoglobin $(\mathrm{HbA} 1 \mathrm{c})$, serum total cholesterol, high-density lipoprotein (HDL) cholesterol and triglycerides. Low-density lipoprotein (LDL) cholesterol was calculated using the Friedewald equation.

\section{Diabetes mellitus}

Diabetes status was determined by the following criteria: primary care records of diagnosis or prescription of diabetes medication, patient recall of diagnosis or taking antidiabetic medication, fasting or oral glucose tolerance test or plasma glucose testing from previous SABRE study visits using the WHO recommendations [22] and those with an $\mathrm{HbAlc}>47 \mathrm{mmol} / \mathrm{mol}$ when tested in the research clinic.

\section{MRI imaging protocol}

All subjects were examined at a single centre, University College Hospital, on a 3 T MRI (Achieva, Philips Healthcare) using an 8-channel phased-array head coil. The protocol included a sagittal T1-weighted 3D-TFE (TR/TE/TI 7/ 3.2/836 ms, flip-angle $18^{\circ}$, voxel size $1 \mathrm{~mm}^{3}$ ) and a transversal 2D pseudo-continuous arterial spin labelling (PCASL) (EPI, TR/TE $4615 / 15 \mathrm{~ms}$, flip-angle $90^{\circ}$, voxel size $3.75 \mathrm{~mm} \times$ $3.75 \mathrm{~mm} \times 5 \mathrm{~mm}, 1-\mathrm{mm}$ slice gap, 20 slices), labelling duration $1800 \mathrm{~ms}$, post-labelling delay $2000 \mathrm{~ms}, 35$ acquisitions, 2 background suppression pulses (1950 ms and $3296 \mathrm{~ms}$ after saturation). There were three repetitions of a proton densityweighted image with TR $=9000 \mathrm{~ms}$ and no background suppression, but otherwise identical parameters as PCASL were also acquired. Planning was aligned to the anterior commissure-posterior commissure line in the transversal plane orthogonal to the T1w sagittal plane, ensuring coverage of the entire cerebrum including the vertex. The labelling plane was positioned on a phase-contrast survey to identify the vessels.

\section{MR segmentation and cerebral blood flow processing}

Tissue segmentation and region labels were obtained using the Geodesic Information Flows framework [23] (https://github. com/KCL-BMEIS/NiftySeg). This method produces a stateof-the-art segmentation and regional labelling by voxel-wise voting between several propagated atlases, guided by the local image similarity. Grey matter and white matter are defined within the propagated atlases. Segmentations of the grey matter, white matter and cerebrospinal fluid space are resampled to the space of the ASL acquisition making use of the known point-spread function to account for down-sampling induced loss of information.

The determination of CBF maps from ASL data followed the simple derived form for PCASL (Eq. 1) from [11] presented in units of $\mathrm{mL} / 100 \mathrm{~g} / \mathrm{min}$ using an open-source in-house software package [24] (https://cmiclab.cs.ucl.ac.uk/CMIC/ NiftyFit-Release). Thirty-five control, (SC) and label (SL) pairs and 5 proton density (SPD) images were averaged to generate single voxel values for the control and label in Eq. 1, where $\lambda$ is the blood/brain partition coefficient $(0.9 \mathrm{~mL} / \mathrm{g})$, PLD is the post-labelling delay between the end of bolus and the start of imaging ( $2000 \mathrm{~ms}), \mathrm{T} 1_{\text {blood }}$ is the blood $\mathrm{T} 1$ relaxation time, $\alpha$ is the labelling efficiency (85\%) and $\tau$ is the labelling duration $(1800 \mathrm{~ms})$. $T 1_{\text {blood }}$ was calculated based on the formula: $\mathrm{T} 1=$ $(0.52 \times \text { Hct }+0.38)^{-1}[13]$ either with fixed value of $43.5 \%$ (corresponding to the standard value of $\mathrm{T} 1=1650 \mathrm{~ms}$ ), which was used in model $1\left(\mathrm{CBF}_{\text {fixed }}\right)$, or calculated based on the Hct values measured from each participant and used in model 2 $\left(\mathrm{CBF}_{\mathrm{Hct}}\right)$. The perfusion difference between $\mathrm{CBF}$ models using a fixed Hct and individualised Hct was calculated as an absolute number and as a percentage of the fixed Hct model.

$$
\begin{aligned}
\mathrm{CBF}= & 6000 \lambda \mathrm{e}^{\mathrm{PLD} / \mathrm{T} \text { lblood }}(\mathrm{SC}-\mathrm{SL}) / \mathrm{SPD} \\
& \left(2 \alpha \mathrm{T} 1_{\text {blood }}\left(1-\mathrm{e}^{-\tau / \mathrm{Tlblood}}\right)\right)[\mathrm{mL} / 100 \mathrm{~g} / \mathrm{min}]
\end{aligned}
$$

Partial volume correction (PVC) was applied based upon the method used in [25]. Results for segmented cortical grey matter PVC CBF are presented. Cortical grey matter CBF without PVC are presented in supplemental material (Supp Table 1).

\section{Statistical analysis}

Analysis was performed using STATA 14.2 (College Station, TX). All analyses were stratified by sex and ethnicity. Continuous data are presented as mean and standard deviation (SD); categorical data are counts and percentages. 
Paired $t$ tests were used to test for statistical significance between the two methods of CBF calculation. Pearson's correlation coefficient was computed to quantify the correlations between Hct and CBF estimates. Comparisons by sex and ethnicity were performed using two-way analysis of variance (ANOVA), followed by individual group-wise comparisons using Fisher's least significant difference test if ANOVA was significant $(p<0.05)$. Multiple linear regression analyses were performed to assess participant characteristic associations with Hct levels with further adjustment for potential confounders, age, mean arterial pressure, diabetes, HbA1C, LDL cholesterol, HDL cholesterol and BMI, chosen a priori. Sample frequency distributions of $\mathrm{CBF}$ calculated with and without correction for individual Hct were analysed using univariate kernel density estimates.

\section{Results}

Five hundred forty-one participants attended MRI. Four hundred ninety-three completed the MRI scan and were successfully processed for CBF. Exclusions were due to susceptibility artefacts such as dental work affecting the radiofrequency label $(n=12)$, claustrophobia/inability to tolerate the scan $(n=9)$, segmentation processing errors due to excessive head movement $(n=25)$, pathology $(n=1)$ and scanner artefact $(n=1)$.

\section{Participant characteristics}

Table 1 shows the basic characteristics of the sample stratified by sex and ethnicity. The sample comprised 493 individuals of whom $40 \%(n=196)$ were women. Women were younger than men (women mean age $\pm \mathrm{SD}, 69.7 \pm 6.4$ years; men $72.9 \pm 5.2$ years; $p<.001)$. White Europeans represented $46 \%$ of the sample, South Asians $35 \%$ and African Caribbeans $19 \%$. A total of $27 \%$ of the participants had type 2 diabetes.

\section{Haematocrit}

The mean (SD) Hct of our sample was $41.6 \%$ (3.7). This is lower than the population mean Hct of $43.5 \%$ suggested in [11], from which a $\mathrm{T} 1_{\text {blood }}$ of $1650 \mathrm{~ms}$ at $3 \mathrm{~T}$ had been calculated. South Asian men had a mean Hct of $42.0 \%$ (3.6), lower than either white European (43.8\% (3.4)) or African Caribbean men (43.0\% (3.5)). Both South Asian $(38.8 \%$ (3.1)) and African Caribbean women (38.8\% (2.4)) had lower mean Hct than white European women (40.6\% (3.0)) (Fig. 1). Age was not associated with Hct $(r=0.06, p=0.2)$.

\section{Cerebral blood flow}

Table 2 shows the results for $\mathrm{CBF}_{\text {fixed }}$ and $\mathrm{CBF}_{\mathrm{Hct}}$ models stratified by sex, ethnicity and diabetes. As a result of correction for individually measured Hct when calculating $\mathrm{T} 1_{\text {blood }}$, $\mathrm{CBF}_{\mathrm{Hct}}$ values were lower than $\mathrm{CBF}_{\text {fixed }}$ values in all categories of sex and ethnicity except for white European and African Caribbean men (Fig. 2).

Figure 3 demonstrates the inverse linear relationship of Hct with $\mathrm{CBF}_{\text {fixed }}$ and $\mathrm{CBF}_{\mathrm{Hct}}$ models. This relationship is attenuated with the use of the $\mathrm{CBF}_{\mathrm{Hct}}$ model although some association of

Table 1 Participant characteristics

\begin{tabular}{|c|c|c|c|c|c|c|c|}
\hline & \multirow[b]{2}{*}{$N$} & \multicolumn{3}{|l|}{ Sex } & \multicolumn{3}{|l|}{ Ethnicity } \\
\hline & & All & Men & Women & White European & South Asian & African Caribbean \\
\hline Sex, $n$ (female) & 493 & 493 (196) & 297 & 196 & $226(78)$ & $175(67)$ & $92(51)^{* *}$ \\
\hline Age, years & 493 & $71.6 \pm 5.9$ & $72.9 \pm 5.2$ & $69.7 \pm 6.4 * *$ & $72.1 \pm 5.8$ & $70.8 \pm 5.6^{* *}$ & $72.0 \pm 6.7$ \\
\hline Diabetes (yes), $n(\%)$ & 493 & $133(27)$ & $80(27)$ & $53(27)$ & $39(17)$ & $65(37)$ & $29(32)^{* *}$ \\
\hline $\mathrm{HbA1c}(\mathrm{mmol} / \mathrm{mol})$ & 486 & $41.7 \pm 9.2$ & $41.2 \pm 8.3$ & $42.5 \pm 10.3$ & $39.2 \pm 7.9$ & $43.9 \pm 8.8^{* *}$ & $43.6 \pm 11.0^{* *}$ \\
\hline BMI $\left(\mathrm{kg} / \mathrm{m}^{2}\right)$ & 489 & $27.5 \pm 4.2$ & $27.1 \pm 3.8$ & $28.2 \pm 4.8^{* *}$ & $27.5 \pm 4.1$ & $26.4 \pm 3.7 * *$ & $29.6 \pm 4.7 * *$ \\
\hline Haematocrit (\%) & 493 & $41.6 \pm 3.7$ & $43.0 \pm 3.5$ & $39.5 \pm 3.0^{* *}$ & $42.7 \pm 3.6$ & $40.8 \pm 3.7 * *$ & $40.7 \pm 3.4^{* *}$ \\
\hline Total cholesterol $(\mathrm{mmol} / \mathrm{L})$ & 492 & $4.7 \pm 1.1$ & $4.5 \pm 1.0$ & $5.1 \pm 1.1 * *$ & $4.8 \pm 1.1$ & $4.5 \pm 1.0 * *$ & $4.8 \pm 1.1$ \\
\hline $\mathrm{LDL}(\mathrm{mmol} / \mathrm{L})$ & 492 & $2.5 \pm 0.9$ & $2.4 \pm 0.9$ & $2.7 \pm 0.9 * *$ & $2.6 \pm 0.9$ & $2.3 \pm 0.9 * *$ & $2.5 \pm 1.0$ \\
\hline HDL (mmol/L) & 492 & $1.60 \pm 0.5$ & $1.5 \pm 0.4$ & $1.8 \pm 0.5^{*}$ & $1.6 \pm 0.5$ & $1.5 \pm 0.4 * *$ & $1.8 \pm 0.6^{* *}$ \\
\hline Diastolic BP (mmHg) & 480 & $79.6 \pm 8.3$ & $79.6 \pm 8.4$ & $79.4 \pm 8.0$ & $79.7 \pm 8.0$ & $78.6 \pm 7.8$ & $80.9 \pm 9.5$ \\
\hline Heart rate $\left(\min ^{-1}\right)$ & 481 & $64.1 \pm 11.1$ & $62.4 \pm 10.9$ & $66.7 \pm 10.9^{* *}$ & $64.5 \pm 10.8$ & $63.4 \pm 11.0$ & $64.7 \pm 12.1$ \\
\hline Mean arterial pressure $(\mathrm{mmHg})$ & 480 & $97.0 \pm 10.0$ & $97.0 \pm 10.0$ & $96.9 \pm 10.0$ & $96.7 \pm 10.1$ & $96.4 \pm 9.9$ & $98.6 \pm 10.0$ \\
\hline Anti-hypertensive medications, (yes) $n(\%)$ & 493 & $283(57)$ & $183(62)$ & $100(51)^{* *}$ & $101(45)$ & $119(68)^{* *}$ & $63(69)^{* *}$ \\
\hline Lipid lowering medications, $n(\%)$ & 493 & $238(48)$ & $138(47)$ & $100(51)$ & $120(53)$ & $64(37)^{* *}$ & $54(59)$ \\
\hline
\end{tabular}

Data are mean \pm standard deviation or number of observations $(n)(\%)$. ${ }^{*} p<0.05, * * p 0.01$ compared to reference (male/white Europeans) by Fisher's LSD test after two-way analysis of variance (ANOVA) 


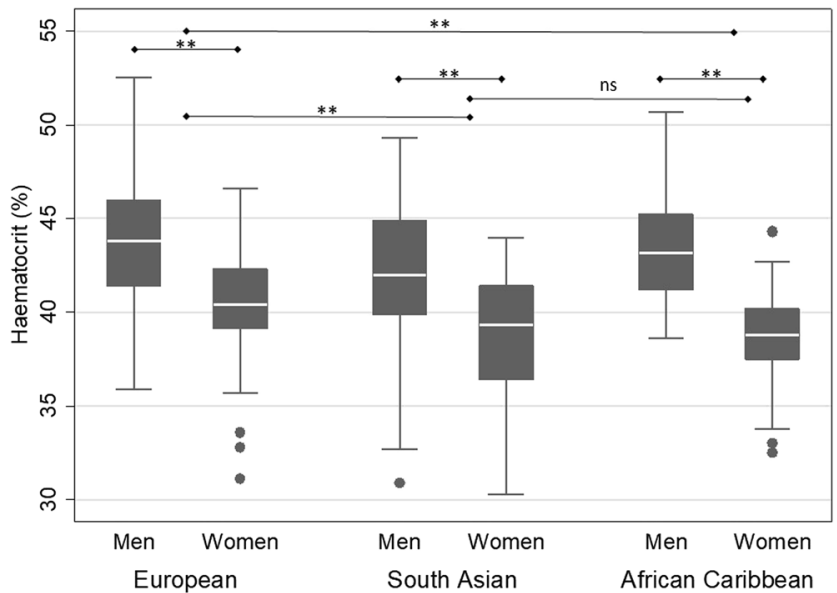

Fig. 1 Boxplot showing median, interquartile range, upper and lower adjacent values and outside values for haematocrit $(\%)$ by sex and ethnicity. $* *=p<0.01$ by two-way analysis of variance followed by Fisher's least significant difference test

Hct with $\mathrm{CBF}$ in men remained $(r=-0.18, p=0.002)$. Further adjustment for potential confounders of mean arterial blood pressure, BMI, diabetes and dyslipidemia did not affect this relationship when entered in a regression model $(\beta=-0.3, \mathrm{CI}-0.6$, $-0.05 \mathrm{~mL} / 100 \mathrm{~g} / \mathrm{min}, p=0.020$ ) (Supp Table 2). (Note: model 2 has fewer subjects due to missing cardiovascular data.)

Introduction of individualised Hct into CBF estimation altered the distributions of cortical CBF values in this elderly population for all ethnicities (Fig. 4). The mean perfusion difference between models was greater for women in all ethnic categories than for men, with a mean decrease for women of

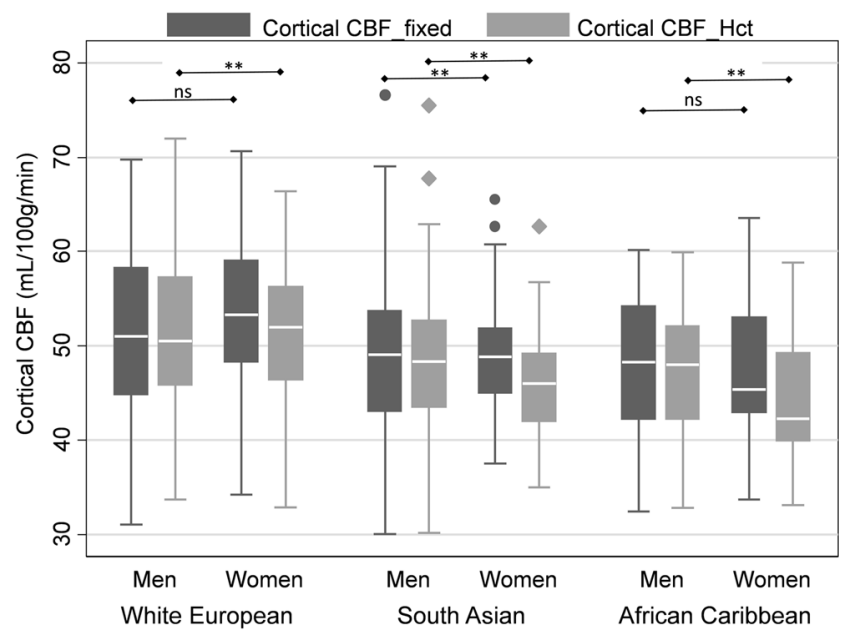

Fig. 2 Boxplot showing median, interquartile range, upper and lower adjacent values and outside values for cerebral blood flow without correction for individual haematocrit $\left(\mathrm{CBF}_{\text {fixed }}\right)$ and cerebral blood flow with correction for individual haematocrit $\left(\mathrm{CBF}_{\mathrm{Hct}}\right)$ by sex and ethnicity. $*=p<0.05, * *=p<0.01$ by two-way analysis of variance followed by Fisher's least significant difference test

$2.7 \mathrm{~mL} / 100 \mathrm{~g} / \mathrm{min}\left(5.4 \%\right.$ of the $\mathrm{CBF}_{\text {fixed }}$ value) $(t=17.1 ; \mathrm{CI}$ $3.0,2.4 \mathrm{~mL} / 100 \mathrm{~g} / \mathrm{min} ; p<0.001, d=0.38$ ). The perfusion difference for African Caribbean women was $-3.1 \mathrm{~mL} /$ $100 \mathrm{~g} / \mathrm{min}\left(6.5 \%\right.$ decrease from the $\mathrm{CBF}_{\text {fixed }}$ value $)(t=10.4$; $\mathrm{CI}-3.6,-2.5 \mathrm{~mL} / 100 \mathrm{~g} / \mathrm{min} ; p<0.001, d=0.48)$, and for South Asian women, it was $-3.0 \mathrm{~mL} / 100 \mathrm{~g} / \mathrm{min}(6.2 \% \mathrm{de}-$ crease from the $\mathrm{CBF}_{\text {fixed }}$ value) $(t=10.9 ; \mathrm{CI}-3.6,-2.5 \mathrm{~mL} /$ $100 \mathrm{~g} / \mathrm{min} ; p<0.001, d=0.56)$. South Asian men were the

Table 2 Comparison of cerebral blood flow without correction for individual haematocrit (CBF_fixed) and cerebral blood flow with correction for individual hemtocrit ( $\mathrm{CBF}$ Hct) by sex, ethnicity and diabetes diagnosis

\begin{tabular}{|c|c|c|c|c|c|c|c|c|c|c|}
\hline & & \multirow[b]{2}{*}{$N$} & \multicolumn{2}{|c|}{$\begin{array}{l}\text { CBF_fixed } \\
(\mathrm{mL} / 100 \mathrm{~g} / \mathrm{min})\end{array}$} & \multicolumn{2}{|c|}{$\begin{array}{l}\text { CBF_Hct } \\
(\mathrm{mL} / 100 \mathrm{~g} / \mathrm{min})\end{array}$} & \multirow{2}{*}{$\begin{array}{l}\text { Difference }(\mathrm{mL} / 100 \mathrm{~g} / \mathrm{min}) \\
\text { Mean }(95 \% \mathrm{CI})\end{array}$} & \multirow{2}{*}{$\begin{array}{l}\text { Mean difference }(\%) \\
\text { Mean }\end{array}$} & \multirow{2}{*}{$p$ value } & \multirow{2}{*}{$\begin{array}{l}\text { Effect size } \\
d\end{array}$} \\
\hline & & & Mean & SD & Mean & $\mathrm{SD}$ & & & & \\
\hline & All & 493 & 50.1 & \pm 7.9 & 48.8 & \pm 7.3 & $-1.3(-1.5,-1.1)$ & -2.6 & $<0.001$ & 0.17 \\
\hline & Men & 297 & 50.0 & \pm 8.3 & 49.6 & \pm 7.6 & $-0.4(-0.7,-0.1)$ & -0.8 & 0.005 & 0.05 \\
\hline & Women & 196 & 50.2 & \pm 7.2 & 47.5 & \pm 6.8 & $-2.7(-3.0,-2.4)$ & -5.4 & $<0.001$ & 0.38 \\
\hline \multirow[t]{3}{*}{ White European } & All & 226 & 51.8 & \pm 8.3 & 51.1 & \pm 7.5 & $-0.7(-1.0,-0.4)$ & -1.4 & $<0.001$ & 0.09 \\
\hline & Men & 148 & 51.1 & \pm 8.6 & 51.1 & \pm 7.7 & $0.0(-0.5,0.4)$ & 0.0 & 0.9 & 0.00 \\
\hline & Women & 78 & 53.2 & \pm 7.6 & 51.1 & \pm 7.0 & $-2.1(-2.6,-1.6)$ & -4.0 & $<0.001$ & 0.29 \\
\hline \multirow[t]{3}{*}{ South Asian } & All & 175 & 49.1 & \pm 7.3 & 47.3 & \pm 6.7 & $-1.8(-2.2,-1.4)$ & -3.7 & $<0.001$ & 0.26 \\
\hline & Men & 108 & 49.4 & \pm 8.1 & 48.4 & \pm 7.3 & $-1.0(-1.5,-0.6)$ & -2.0 & $<0.001$ & 0.14 \\
\hline & Women & 67 & 48.7 & \pm 5.8 & 45.7 & \pm 5.0 & $-3.0(-3.6,-2.5)$ & -6.2 & $<0.001$ & 0.56 \\
\hline \multirow[t]{3}{*}{ African Caribbean } & All & 92 & 47.6 & \pm 6.7 & 45.8 & \pm 6.3 & $-1.8(-2.3,-1.3)$ & -3.8 & $<0.001$ & 0.29 \\
\hline & Men & 41 & 47.7 & \pm 7.0 & 47.4 & \pm 6.4 & $-0.3(-0.9,0.3)$ & -0.6 & 0.3 & 0.04 \\
\hline & Women & 51 & 47.6 & \pm 6.6 & 44.5 & \pm 6.0 & $-3.1(-3.6,-2.5)$ & -6.5 & $<0.001$ & 0.48 \\
\hline Without diabetes & & 377 & 50.5 & \pm 7.8 & 49.4 & \pm 7.2 & $-1.1(-1.4,-0.8)$ & -2.2 & $<0.001$ & 0.15 \\
\hline With diabetes & & 116 & 49.0 & \pm 8.3 & 47.2 & \pm 7.5 & $-1.8(-2.3,-1.4)$ & -3.7 & $<0.001$ & 0.23 \\
\hline
\end{tabular}

Data are mean \pm standard deviation, except difference $(95 \%$ confidence interval CI), mean difference (\%). $p$ values were calculated using a Student's $t$ test. Effect size is Cohen's $d$ 

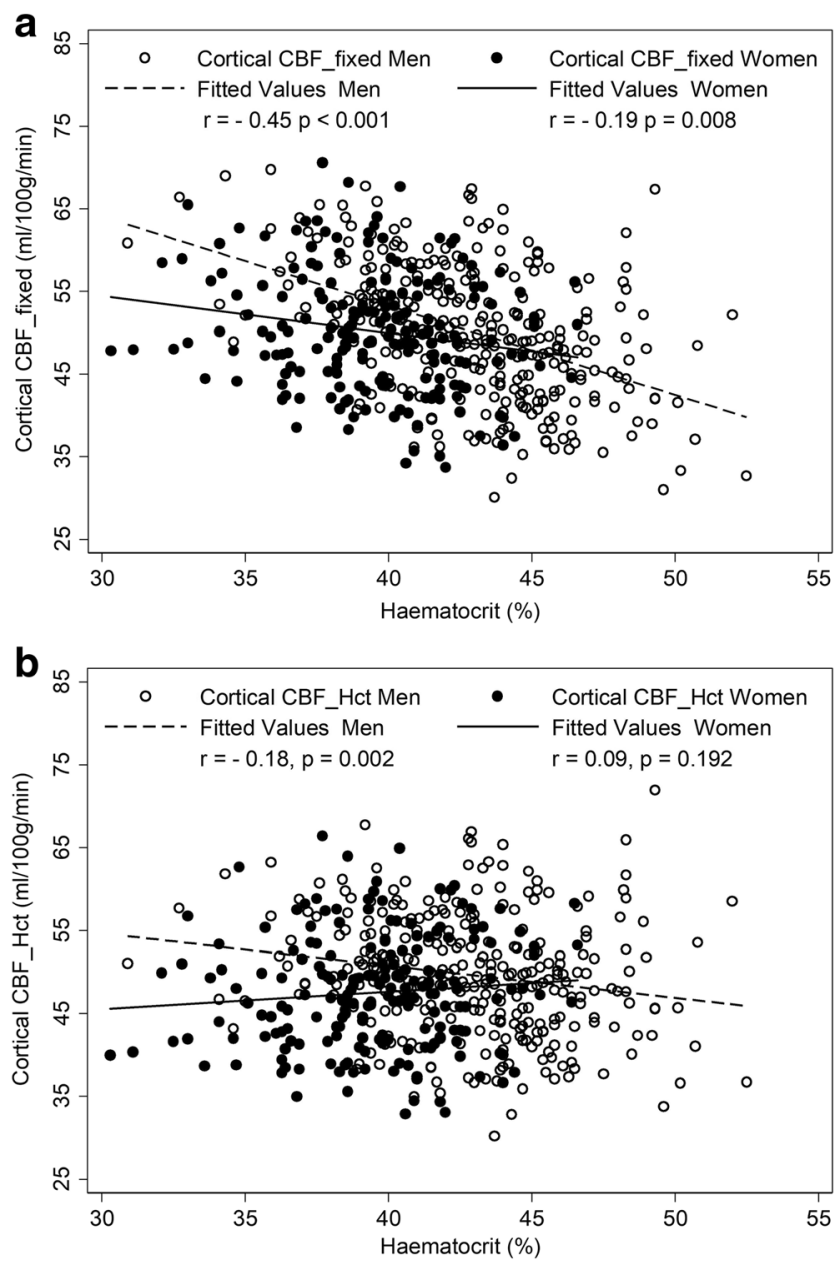

Fig. 3 Scatterplots showing the effect of correction for individual haematocrit on the correlation between haematocrit and cortical cerebral blood flow in men and women. Fixed Hct $\left(\mathrm{CBF}_{\text {fixed }}\right)(\mathbf{a})$; individualised Hct $\left(\mathrm{CBF} \_\right.$Hct $)(\mathbf{b})$
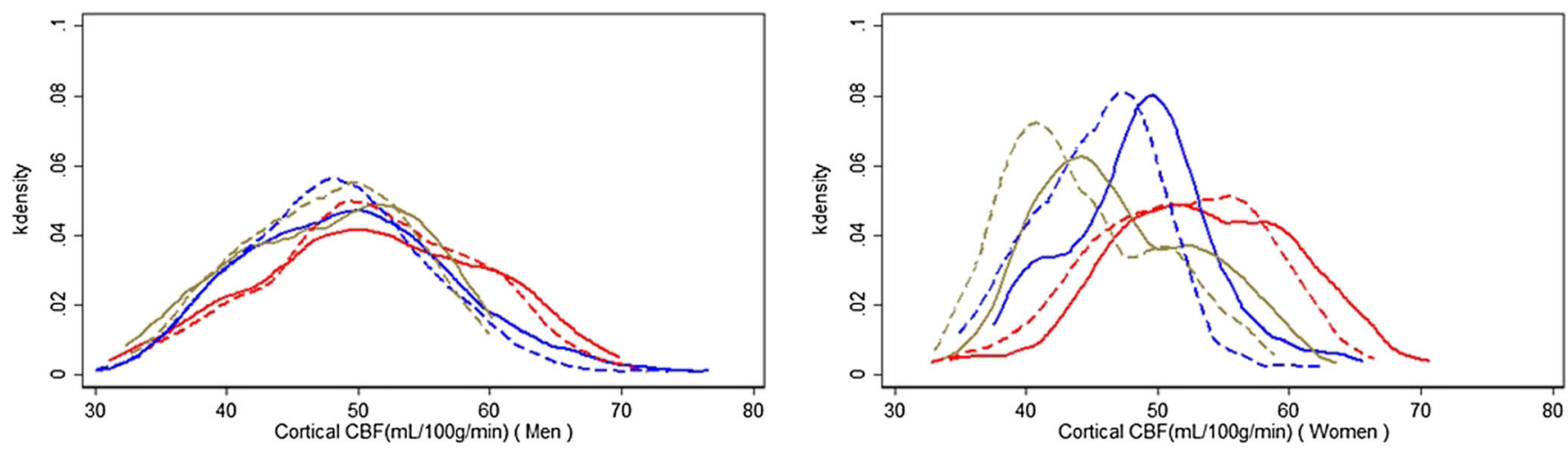

People with diabetes had a mean (SD) Hct of $40.7 \%$ (4.1) compared with $42.0 \%$ (3.5) for those without diabetes. The perfusion difference between CBF models of people with diabetes was $-1.8 \mathrm{~mL} / 100 \mathrm{~g} / \mathrm{min}\left(3.7 \%\right.$ of the $\mathrm{CBF}_{\text {fixed }}$ value) $(t=7.8$; CI $-2.3,-1.4 \mathrm{~mL} / 100 \mathrm{~g} / \mathrm{min} ; p<0.001, d=0.23)$, whereas those without diabetes only displayed a difference of $-1.1 \mathrm{~mL} / 100 \mathrm{~g} / \mathrm{min}\left(2.2 \%\right.$ of the $\mathrm{CBF}_{\text {fixed }}$ value $)(t=8.4 ; \mathrm{CI}$ $-1.4,-0.8 \mathrm{~mL} / 100 \mathrm{~g} / \mathrm{min}, p<0.001, d=0.15$ ) (Fig. 6).

\section{Discussion}

This study has shown that Hct levels differ according to sex and ethnicity and that this influences CBF estimated from ASL. Importantly, failure to adjust $\mathrm{T} 1_{\text {blood }}$ according to sex and ethnic variation in Hct leads to a significant overestimation of $\mathrm{CBF}$ in women and non-European populations. Although other studies have investigated the effect of Hct on CBF estimation with ASL in sickle cell [26] and neonatal [27]

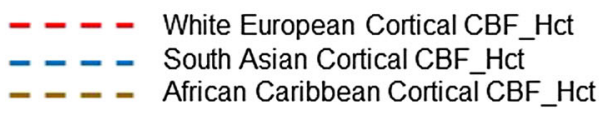

Fig. 4 Kernel density (kdensity) plots of $\mathrm{CBF}$ without correction for individual haematocrit $\left(\mathrm{CBF}_{\text {fixed }}\right)$ and $\mathrm{CBF}$ with correction for individual haematocrit $\left(\mathrm{CBF}_{\mathrm{Hct}}\right)$ by sex and ethnicity 
Fig. 5 CBF maps overlaid on segmented $\mathrm{T} 1 \mathrm{w}$ without and with adjustment for measured haematocrit. The subject was a white European woman with an haematocrit of $37.3 \%$

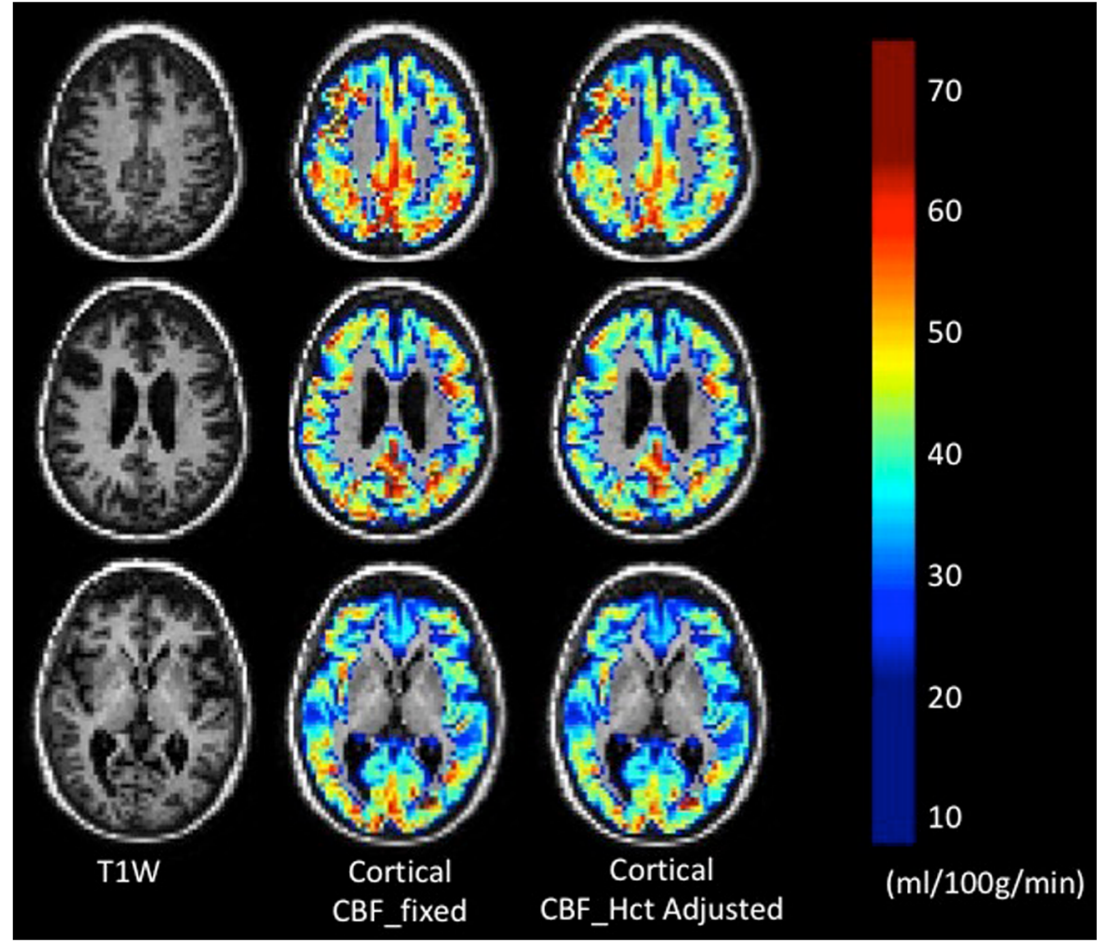

groups; this is to the best of our knowledge, the first study to address this in a community-based elderly multi-ethnic population.

A study by Parkes et al [28] using continuous ASL found females had higher grey matter $\mathrm{CBF}$ than males by $13 \%$. It seems likely that failure to measure individual Hct influenced these observations. We found a similar difference in $\mathrm{CBF}$ between white European men and women $(+9.1 \%$ for women) when neither Hct nor PVC was accounted for, but this difference was completely abolished by correction for Hct and PVC. Following correction for PVC and Hct, CBF was

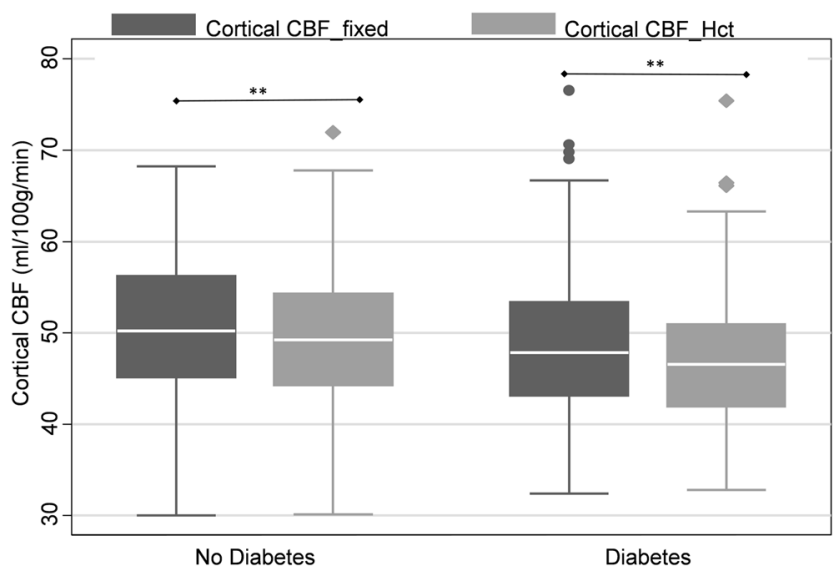

Fig. 6 Boxplot showing median, interquartile range, upper and lower adjacent values and outside values for CBF without correction for individual haematocrit ( $\mathrm{CBF}$ fixed) and $\mathrm{CBF}$ with correction for individual haematocrit $\left(\mathrm{CBF}_{\mathrm{Hct}}^{-}\right)$by diabetes status. $*=p<0.05$, $* *=p<0.01$ by Student's $t$ test slightly lower for women than for men in South Asian $(-5.6 \%)$ and African Caribbean $(-6.0 \%)$ ethnicities. This finding is broadly aligned with an ${ }^{15} \mathrm{O}$ PET study where gender differences in $\mathrm{CBF}$ evident in younger subjects were not significant in subjects older than 65 years [29]. It has been suggested that gender differences in young subjects are due to high oestrogen levels in females causing CBF fluctuations during the menstrual cycle [30]. Our large elderly sample scanned with age-appropriate parameters such as a long PLD and with CBF calculated using individualised values is likely to provide a more accurate reflection of true physiological differences than studies that have used a 'one size fits all' protocol.

We also demonstrated that the utilisation of individually measured Hct in the calculation of $\mathrm{T}_{1}$ blood reduced the inverse correlation between $\mathrm{CBF}$ and Hct. However, some association remained in men even after adjustment for mean arterial blood pressure, diabetes and dyslipidemia. Increased CBF is an expected response to decreases in haemoglobin (and thus Hct) as a mechanism to sustain the cerebral metabolic rate of oxygen levels $\left(\mathrm{CMRO}_{2}\right)$ as demonstrated by Ibaraki et al [31]. It has also been suggested that higher Hct levels and concomitant increases in blood viscosity may influence capillary flow due to alterations in functional shunting [32]. The higher Hct levels found in men may provide an explanation why we found some association between Hct and CBF only in men as increasing Hct increases blood viscosity in a non-linear relationship, and it has been suggested that increased viscosity decreases CBF [33]. 
Lower values of Hct in those with diabetes indicate that further investigation using larger samples is warranted to investigate the interactions of sex, ethnicity, Hct and the effect of diabetes on $\mathrm{CBF}$.

Investigation of the association of age and CBF, which had yielded conflicting evidence in previous studies [10, 28, 34], was not the main aim of our study. However, it is noteworthy that we found an association between age and CBF in the non-partial volume-corrected data which disappeared following partial volume correction. A likely explanation for this is the decreased brain volume in older subjects which makes the ASL perfusion data more prone to partial volume effects with non-perfused CSF, thereby leading to an artificial CBF decrease. However, PVC may introduce overestimation of cortical $\mathrm{CBF}$ particularly in examinations hampered by head movement or poor SNR, either in the T1 structural images used for segmentation or in the ASL acquisition which as a subtraction technique is particularly susceptible to movement artefact. Some movement inevitably remains in a large population study of elderly individuals and this may be a potential source of under- or overestimation of partial volume-corrected $\mathrm{CBF}$ in our cohort.

Despite a more diverse ethnic sample, our cortical grey matter $\mathrm{CBF}$ values are comparable with previous studies using PCASL on elderly, community-dwelling populations [6, 7]. PCASL has some limitations when applied to elderly subjects. One factor affecting CBF quantification is blood velocity relative to the labelling plane. Any discrepancies from the expected range of blood velocities due to vascular pathologies such as internal carotid stenosis or vessel tortuosity might result in reduced labelling efficiency and therefore CBF underestimation. Our study used 2D PCASL, rather than a 3D technique, which may be liable to decreased SNR due to longer T1 blood relaxation in slices near the vertex due to sequential slice acquisition [35], despite using slice-timing correction. Inefficiency of background suppression pulses during multiple slice acquisitions may also have affected the ASL signal.

The main strength of this study was its large communitybased, elderly, ethnically diverse sample studied in a single centre so that all protocols were consistently applied throughout. Limitations of the study include a potential selection bias towards healthy individuals who were willing and able to attend the research clinic. Another limitation was the absence of direct $\mathrm{T} 1_{\text {blood }}$ measurements, although this was mitigated by the use of a previously published model to account for the effect of Hct [13]. Hct is the main determinant of $\mathrm{T} 1_{\text {blood }}$, but other factors such as serum ferritin and HDL cholesterol may also contribute to a minor extent [36]. Measurement of arterial rather than venous Hct would have been more appropriate to estimate CBF. However, measuring arterial Hct in clinic was impractical and it was considered that venous and arterial Hct were closely related and importantly that venous Hct could be sampled consistently [37]. Although $\mathrm{T} 1_{\text {blood }}$ can be easily directly measured in the left ventricle and has lower test-retest variability compared with measured Hct [38], comparable sequences in the brain are more challenging due to partial volume effects, blood velocity and pulsatility in the measured vessel $[27,39]$.

Our findings suggest that research studies using ASL to measure $\mathrm{CBF}$ should routinely measure Hct to adjust $\mathrm{T} 1_{\text {blood }}$, especially in inhomogenous samples. Alternatively, substitution of an appropriate gender- and ethnicity-specific Hct value derived from population group means or direct measurement of $\mathrm{T}_{\text {blood }}$ during MRI would improve accuracy of CBF measurement. Further research is warranted into whether adjustment to the Hct value in CBF models to accommodate demographic and pathological differences provides stronger associations with cerebrovascular disease, dementia and cognitive decline than previous models using a fixed mean Hct value. Results from previous studies may need to be interpreted with caution where there are ethnic, gender and pathological differences in the sample. Such an approach may improve early risk assessment in ethnic groups and identify potentially vulnerable groups such as those with known vascular or metabolic disease.

In conclusion, we demonstrated that $\mathrm{CBF}$ values obtained from ASL using a fixed Hct mean may lead to systematic errors, resulting most frequently in an overestimation of CBF in female subjects and non-Caucasian ethnicities. It is important to be aware of this when using CBF threshold values to assess disease status or severity. This applies not only to the discrimination between normal ageing and a neurogenerative disease such as $\mathrm{AD}$ but could potentially also influence the discrimination between high- and low-grade brain tumours or determination of penumbral threshold values in stroke. We therefore argue that whenever possible, individualised measures of Hct should be included in CBF calculations by ASL.

Acknowledgements Data used in preparation of this article were obtained from the SABRE Study (www.sabrestudy.org).

Funding This study has received funding from the British Heart Foundation, Diabetes UK, the Medical Research Council and the Wellcome Trust. The current visit (visit 3) was funded by the British Heart Foundation (CS/13/1/30327). This study was supported by the National Institute for Health Research University College London Hospitals Biomedical Research Centre.

FB is supported by the NIHR biomedical research centre at UCLH.

DO is supported by the EPSRC-funded UCL Centre for Doctoral Training in Medical Imaging (EP/L016478/1) and the Wolfson Foundation.

\section{Compliance with ethical standards}

Guarantor The scientific guarantor of this publication is Prof Nish Chaturvedi.

Conflict of interest The authors of this manuscript declare no relationships with any companies, whose products or services may be related to the subject matter of the article.

Statistics and biometry No complex statistical methods were necessary for this paper. 
Informed consent Written informed consent was obtained from all subjects (patients) in this study.

Ethical approval Institutional Review Board approval was obtained.

\author{
Methodology \\ - prospective \\ - cross-sectional study \\ - performed at one institution
}

Open Access This article is distributed under the terms of the Creative Commons Attribution 4.0 International License (http:// creativecommons.org/licenses/by/4.0/), which permits unrestricted use, distribution, and reproduction in any medium, provided you give appropriate credit to the original author(s) and the source, provide a link to the Creative Commons license, and indicate if changes were made.

\section{References}

1. Haller S, Zaharchuk G, Thomas DL et al (2016) Arterial spin labeling perfusion of the brain: emerging clinical applications. Radiology 281:337-356

2. Bastos-Leite AJ, Kuijer JP, Rombouts SA et al (2008) Cerebral blood flow by using pulsed arterial spin-labeling in elderly subjects with white matter hyperintensities. AJNR Am J Neuroradiol 29: 1296-1301

3. Leeuwis AE, Benedictus MR, Kuijer JP et al (2016) Lower cerebral blood flow is associated with impairment in multiple cognitive domains in Alzheimer's disease. Alzheimers Dement 13(5):531540

4. Schuff N, Matsumoto S, Kmiecik J et al (2009) Cerebral blood flow in ischemic vascular dementia and Alzheimer's disease, measured by arterial spin-labeling magnetic resonance imaging. Alzheimers Dement 5:454-462

5. Benedictus MR, Leeuwis AE, Binnewijzend MA et al (2017) Lower cerebral blood flow is associated with faster cognitive decline in Alzheimer's disease. Eur Radiol 27:1169-1175

6. Binnewijzend MA, Benedictus MR, Kuijer JP et al (2016) Cerebral perfusion in the predementia stages of Alzheimer's disease. Eur Radiol 26:506-514

7. Binnewijzend MA, Kuijer JP, Benedictus MR et al (2013) Cerebral blood flow measured with 3D pseudocontinuous arterial spinlabeling MR imaging in Alzheimer disease and mild cognitive impairment: a marker for disease severity. Radiology 267:221-230

8. Binnewijzend MA, Kuijer JP, van der Flier WM et al (2014) Distinct perfusion patterns in Alzheimer's disease, frontotemporal dementia and dementia with Lewy bodies. Eur Radiol 24:23262333

9. Henriksen OM, Kruuse C, Olesen J et al (2013) Sources of variability of resting cerebral blood flow in healthy subjects: a study using ${ }^{133}$ Xe SPECT measurements. J Cereb Blood Flow Metab 33: 787-792

10. Clement P, Mutsaerts H-J, Václavů L et al (2018) Variability of physiological brain perfusion in healthy subjects-a systematic review of modifiers. Considerations for multi-center ASL studies. J Cereb Blood Flow Metab 38:1418-1437

11. Alsop DC, Detre JA, Golay X et al (2015) Recommended implementation of arterial spin-labeled perfusion MRI for clinical applications: a consensus of the ISMRM perfusion study group and the European consortium for ASL in dementia. Magn Reson Med 73: $102-116$
12. Buxton RB (2005) Quantifying CBF with arterial spin labeling. J Magn Reson Imaging 22:723-726

13. Lu H, Clingman C, Golay X et al (2004) Determining the longitudinal relaxation time (T1) of blood at 3.0 Tesla. Magn Reson Med 52:679-682

14. de Simone G, Devereux RB, Chien S et al (1990) Relation of blood viscosity to demographic and physiologic variables and to cardiovascular risk factors in apparently normal adults. Circulation 81: 107-117

15. Cheng CK-W, Chan J, Cembrowski GS et al (2004) Complete blood count reference interval diagrams derived from NHANES III: stratification by age, sex, and race. Lab Hematol 10:42-53

16. Lim E, Miyamura J, Chen JJ (2015) Racial/ethnic-specific reference intervals for common laboratory tests: a comparison among Asians, Blacks, Hispanics, and White. Hawaii J Med Public Health $74: 302$

17. McDonough J, Garrison G, Hames C (1964) Blood pressure and hypertensive disease among negroes and whites: a study in Evans County, Georgia. Ann Intern Med 61:208-228

18. Tamariz LJ, Young JH, Pankow JS et al (2008) Blood viscosity and hematocrit as risk factors for type 2 diabetes mellitus: the atherosclerosis risk in communities (ARIC) study. Am J Epidemiol 168: $1153-1160$

19. Vlagopoulos PT, Tighiouart H, Weiner DE et al (2005) Anemia as a risk factor for cardiovascular disease and all-cause mortality in diabetes: the impact of chronic kidney disease. J Am Soc Nephrol 16: 3403-3410

20. de Jager J, Kooy A, Lehert P et al (2010) Long term treatment with metformin in patients with type 2 diabetes and risk of vitamin B-12 deficiency: randomised placebo controlled trial. BMJ 340:c2181

21. Tillin T, Forouhi NG, McKeigue PM et al (2012) Southall And Brent REvisited: cohort profile of SABRE, a UK populationbased comparison of cardiovascular disease and diabetes in people of European, Indian Asian and African Caribbean origins. Int J Epidemiol 41:33-42

22. World Health Organisation (1999) Definition, diagnosis and classification of diabetes mellitus and its complications: report of a WHO consultation. Part 1, Diagnosis and classification of diabetes mellitus. World Health Organization, Geneva. Available via https://apps.who. int/iris/bitstream/handle/10665/66040/WHO_NCD_NCS_99.2.pdf; jsessionid=5CE4EC0 166DD7CAF6D 55 C4FE363044DE? sequence $=1$. Accessed 24 Dec 2018

23. Cardoso MJ, Modat M, Wolz R et al (2015) Geodesic information flows: spatially-variant graphs and their application to segmentation and fusion. IEEE Trans Med Imaging 34:1976-1988

24. Melbourne A, Toussaint N, Owen D et al (2016) NiftyFit: a software package for multi-parametric model-fitting of $4 \mathrm{D}$ magnetic resonance imaging data. Neuroinformatics 14:319-337

25. Asllani I, Borogovac A, Brown TR (2008) Regression algorithm correcting for partial volume effects in arterial spin labeling MRI. Magn Reson Med 60:1362-1371

26. Vaclavu L, van der Land V, Heijtel DF et al (2016) In vivo T1 of blood measurements in children with sickle cell disease improve cerebral blood flow quantification from arterial spin-labeling MRI. AJNR Am J Neuroradiol 37:1727-1732

27. De Vis JB, Hendrikse J, Groenendaal F et al (2014) Impact of neonate haematocrit variability on the longitudinal relaxation time of blood: implications for arterial spin labelling MRI. Neuroimage Clin 4:517-525

28. Parkes LM, Rashid W, Chard DT et al (2004) Normal cerebral perfusion measurements using arterial spin labeling: reproducibility, stability, and age and gender effects. Magn Reson Med 51:736743

29. Aanerud J, Borghammer P, Rodell A et al (2017) Sex differences of human cortical blood flow and energy metabolism. J Cereb Blood Flow Metab 37:2433-2440 
30. Baxter LR, Mazziotta JC, Phelps ME et al (1987) Cerebral glucose metabolic rates in normal human females versus normal males. Psychiatry Res 21:237-245

31. Ibaraki M, Shinohara Y, Nakamura K et al (2010) Interindividual variations of cerebral blood flow, oxygen delivery, and metabolism in relation to hemoglobin concentration measured by positron emission tomography in humans. J Cereb Blood Flow Metab 30:12961305

32. Ostergaard L, Engedal TS, Moreton F et al (2016) Cerebral small vessel disease: capillary pathways to stroke and cognitive decline. J Cereb Blood Flow Metab 36:302-325

33. Grotta J, Ackerman R, Correia J et al (1982) Whole blood viscosity parameters and cerebral blood flow. Stroke 13:296-301

34. Chen JJ, Rosas HD, Salat DH (2011) Age-associated reductions in cerebral blood flow are independent from regional atrophy. Neuroimage 55:468-478
35. Dolui S, Vidorreta M, Wang Z et al (2017) Comparison of Pasl, Pcasl, and background-suppressed $3 \mathrm{~d}$ Pcasl in mild cognitive impairment. Hum Brain Mapp 38:5260-5273

36. Rosmini S, Bulluck H, Treibel TA et al (2016) Hematocrit, iron and HDL-cholesterol explain $90 \%$ of variation in native blood T1. J Cardiovasc Magn Reson 18:086

37. Mokken FC, van der Waart FJ, Henny CP et al (1996) Differences in peripheral arterial and venous hemorheologic parameters. Ann Hematol 73:135-137

38. Treibel TA, Fontana M, Maestrini V et al (2016) Automatic measurement of the myocardial interstitium: synthetic extracellular volume quantification without hematocrit sampling. JACC Cardiovasc Imaging 9:54-63

39. Li W, Liu P, Lu H et al (2017) Fast measurement of blood T1 in the human carotid artery at 3T: accuracy, precision, and reproducibility. Magn Reson Med 77:2296-2302

Publisher's note Springer Nature remains neutral with regard to jurisdictional claims in published maps and institutional affiliations. 